\title{
The Effects of Atmospheric Pressure Fluctuations on Human Behaviour Related to Injury Occurrences: Study on the Background of Low and Moderate Levels of Geomagnetic Activity
}

\author{
Lyudmyla Didyk, ${ }^{1}$ Yuriy Gorgo, ${ }^{2}$ Alina Prigancova, ${ }^{3}$ Igor Tunyi, ${ }^{3}$ Magdalena Vaczyova, ${ }^{3}$ \\ Sergey Mamilov, ${ }^{2}$ and Joris Dirckx ${ }^{4}$ \\ ${ }^{1}$ Institute of Physics, National Academy of Sciences of Ukraine, Prospekt Nauki, 46, Kyiv 03028, Ukraine \\ ${ }^{2}$ Institute of Applied Problems of Physics and Biophysics, National Academy of Sciences of Ukraine, Slugbova Street, 3 , \\ Kyiv 03142, Ukraine \\ ${ }^{3}$ Geophysical Institute, Slovak Academies of Sciences, Dubravska cesta 9, 84528 Bratislava, Slovakia \\ ${ }^{4}$ Laboratory of Biomedical Physics, University of Antwerp, Groenenborgerlaan, 171, 2020 Antwerp, Belgium \\ Correspondence should be addressed to Lyudmyla Didyk, la_didyk@iop.kiev.ua
}

Received 7 December 2011; Accepted 10 January 2012

Academic Editor: U. Kulshrestha

Copyright ( $\odot 2012$ Lyudmyla Didyk et al. This is an open access article distributed under the Creative Commons Attribution License, which permits unrestricted use, distribution, and reproduction in any medium, provided the original work is properly cited.

\begin{abstract}
The effects of slight atmospheric pressure fluctuations (APFs) within two ranges of periods attributed mostly to far infrasound ( $3 \mathrm{~s}-$ $120 \mathrm{~s}$ ) and internal gravity waves (120s-1200s) on human behaviour related to injury occurrences, within the one-year interval, are examined. Special interest is paid to the analysis of combined effects of APFs and geomagnetic activity (GMA) attributed to low and moderate levels. The relations between the daily number of emergency transport events due to sport injuries (EEI) and daily mean of APF integral amplitudes within the two ranges (DHAI and DHAG, resp.) along with the planetary geomagnetic index Ap are analysed using the regression models based on categorization. As shown, the high DHAI is a rather strong meteorotropic factor, being of relevance to increase in the incidence of sport injuries. The high DHAG appears to be of opposite sense on the background of low DHAI, promoting the decreased EEI number. The consideration of combined effects of the APF and GMA reveals that the negative effects of high DHAI are more pronounced in combination with low Ap levels. The results are discussed from the viewpoint of the necessity of further medico-meteorological studies using databases of most disturbed geophysical conditions.
\end{abstract}

\section{Introduction}

Numerous studies in medical meteorology indicate that abrupt daily variations in the atmospheric pressure (AP) are important meteorotropic factor rendering adverse effects on health and different kind of human activity. However, insufficient attention is paid to the other bioeffective physical characteristics of AP.

It is known, that variety of atmospheric events make pressure fluctuate in very wide range of periods. Particular interest is the meteorotropic features of the atmospheric pressure fluctuations (APF) in the infrasound frequency range $(0.003 \mathrm{~Hz}<f<1 \mathrm{~Hz})$ related to natural noises in the atmosphere [1-3]. Most powerful source of APF in stormy weather is the chaotic turbulent airflows induced by strong wind $[4,5]$. Apart from acoustic waves produced by air compressibility, the internal gravity waves (IGWs) generated by vertical density stratification considerably contribute to the atmospheric noise. Their periods near the Earth surface are within the range of a minute up to several hours $[2,4,6]$. Below the frequency of $0.003 \mathrm{~Hz}$, the atmospheric waves are transformed into almost pure IGW. It is believed that IGWs with periods between $1 \mathrm{~min}$ and $40 \mathrm{~min}$ are of relevance to the human reactions [7]. A varying number of sources generate IGWs at lower level of the atmosphere including convective and frontal activity, wind shear, and topography. Severe weather conditions, such as frontal activity, monsoon, thunderstorms, and hurricanes, as well as more intense 
weather phenomena (typhoons, tornados, cyclones, ets.), are accompanied by generation of acoustical and acousticgravity waves.

The important feature of the APF is that they penetrate buildings [3, 7] and, therefore, could be responsible for weather sensitivity symptoms not only outdoors, but also indoors. Long ago Mezernitsky [8] emphasized that rapid "micropulsations" of atmospheric pressure are capable of influencing human organisms in the worst way. The adverse effects of infrasonic waves, generated by severe storm activity on selected kinds of human behaviour, particularly the increase in the automobile accidents reported Green and Dunn $[1]$.

It has also been speculated that the heightened anxiety levels in people with mental disorders increase in suicide incidence and the more frequent occurrences of cardiac arrhythmias on days with strong wind are likely, at least partly, to be due to some biological response to wind-generated rapid atmospheric pressure perturbations $[2,9,10]$. The effects of winds blowing from the mountains on human mental activity, characterized by parameters such as reaction time and/or duration of active attention, and indirect indications such as the behaviour resulting in traffic accidents were considered in a number of studies [11-13]. Lee and Garraway [14] found a significant effect of wind strength on the risk of sport injuries.

Our previous study revealed meteorotropic effects of high APF in the far infrasound range concerning people with circulatory system diseases [3]. The purpose of this study is to examine whether the high APF in the far infrasound and in IGW range of periods can influence human behaviour related to injury occurrences. Sports activity is chosen, as it is a relevant area to the high risk of injury due to extraordinary inner strain up to the limits of human endurances. Therefore, the possible relations of APF within the two ranges of periods ( $3 s-120 s$ and $120 s-1200 s$ ) with the daily number of emergency transport events due to sport injuries (EEI) are analysed allowing for the confounding effects of basic meteorological parameters. In addition, the possible association of the APF effects with wind velocity is verified. Special interest is paid to the analysis of combined effects of APF and geomagnetic activity (GMA), since there is increasing amount of evidence indicating biological effects of geomagnetic conditions.

\section{Materials and Methods}

2.1. Data on APF and Relevant Natural Physical Variables. The study was conducted in Kyiv city (Ukraine). The continuous measurements of atmospheric pressure, carried out every $0.5 \mathrm{~s}$ with a standard high-sensitive ( $1 \mathrm{~Pa}$ ) microbarometer (Atmosphere-1, Industrial science-technical cooperative "Dobry shlyah") outdoors during the same one-year period (from 1 July 2005 to 30 June 2006) as in our previous study [3], are a database for the analysis. The APF spectral parameters and average hourly integral amplitude (HA) in two ranges of periods (HAI: $3 s-120 s$ and HAG: $120 s-1200 s$ ) were calculated with a special computer program developed by us using Matlab software. According to known definitions
$[4,6,9]$, the APF periods are related primarily to the far infrasound in the first range (thereafter I-range) and to IGWs in the second range (thereafter G-range).

Three hourly meteorological data on temperature, relative humidity, wind velocity, and atmospheric pressure were received from the Kyiv Geophysical Observatory. Data on planetary geomagnetic activity (Ap index) are available at the Internet (World Data Centre for Geomagnetism, Kyoto).

\subsection{Database on Sport Injuries, Its Processing, and Statistical} Analysis. The database on daily EEI number according to ICD-10 coding rules was obtained from the Kyiv Station of emergency services and medicine of catastrophes. The database includes only the total number of EEI related to professional and amateur sport activities regardless of the kind of sport activity or the severity of injuries.

Within the preprocessing of the EEI one-year database, all public holidays were excluded from the data. Since the EEI and atmospheric data for a few days were not available, altogether 345 days were used for the analysis when 1533 EEI took place. The EEI number proved to be larger on Saturdays than on other days of the week, although the significant difference for EEI was revealed only between Saturdays and Wednesdays $(P<0.05)$. The effect of the day of the week was controlled for by dummy variables.

The EEI number was significantly less in summer than for other seasons of the year $(P<0.00001)$ for which the EEI numbers are comparable. The decreased EEI number in summer seems to be associated with the peculiarities of social schedules (i.e., a holiday season when amateur sports and school sport activities are minimized). The number of a successive day in the course of the year (ND) was included in the model as the potential confounding variable to control for the temporal trend in the annual EEI pattern. The introduced ND variable allows taking into account not only the effects of seasonal changes of natural physical environmental conditions, but also peculiarities of the social background (e.g., obligatory schedules usual for sport activities, summer holidays, etc.).

The EEI number was related to the daily mean of HAI (DHAI) and HAG (DHAG). Since the APFs are causally related to the wind-induced turbulence, the additional analysis of possible association of the DHA effects with wind velocity $(\mathrm{WV})$ was performed in detail. The possible relation between the EEI and geomagnetic activity (GMA), as well as combined effects of APF and GMA, was examined using the daily planetary geomagnetic Ap index [15]. This parameter is widely used in biomedical investigations. According to the previous investigations, Ap index is the effective parameter for human psychophysiological and autonomic reactions [16-18]. On the other hand, the Ap index is considered to be an appropriate criterion to identify the degree of GMA at the mid-latitudes, describing quiet $(0<\mathrm{Ap}<8)$, unsettled $(8<$ Ap $<16)$, active $(16<\mathrm{Ap}<30)$ geomagnetic conditions, and three levels of geomagnetic disturbances (minor $(30<\mathrm{Ap}<$ $50)$, major $(50<\mathrm{Ap}<100)$, and severe $(100<\mathrm{Ap})$ storms, resp.) [19]. Their duration in the studied one-year interval is 190, 99, 40, 10, 4 and 2 days, respectively. 
TABLE 1: 95\% Confidence interval (CI) of DHAI, DHAG, WV, and Ap values, their maximum and minimum values calculated within the one-year interval analysed.

\begin{tabular}{lccc}
\hline & $95 \%$ CI & Maximum & Minimum \\
\hline DHAI $(\mathrm{Pa})$ & $2.65-3.06$ & 11.07 & 0.70 \\
DHAG $(\mathrm{Pa})$ & $2.27-2.50$ & 10.34 & 0.88 \\
WV $(\mathrm{m} / \mathrm{s})$ & $2.15-2.37$ & 5.50 & 0.00 \\
Ap & $8.74-11.23$ & 101.87 & 0.00 \\
\hline
\end{tabular}

The mean daily values of temperature, relative humidity, and atmospheric pressure were considered as potential confounding meteorological variables.

A polynomial approximation was used to visually assess the functional form of the relationship between the EEI and the independent variable. The regression models based on categorisation $[3,20]$ were applied to explore the relations of EEI with independent variables. The advantage of such approach over parametric regression is the ability to reveal threshold effects, while the strong assumption about the relationships is not required. At first, the values of the independent variables were divided into quartiles. If a significantly different EEI values were revealed only between two groups of the quartiles, then the independent variable values were divided only into two categories. Because of the susceptibility of categorical methods for changing boundaries of the categories, the number of days for these two categories was defined as $n 1$ and $n 2$, respectively, as soon as a tested boundary between them has revealed the most significant difference for the EEI number. The nonparametric smoothing procedures (Loess technique) were used to eliminate (in a stepwise manner) the confounding variable patterns in the EEI data.

All independent variables, as well as the EEI number are not normally distributed. Therefore, non-parametric (Mann-Whitney $U$-test and Sherman rank correlation test) estimations were applied. Statistical analysis was performed with Matlab 6.6 (Curve Fitting Toolbox), Statistica 6, and MS Excell.

\section{Results}

3.1. Natural Physical Characteristics. Descriptive statistics of variables DHA, WV, and Ap (Table 1) indicate the moderate level of atmospheric activity within the one-year interval analysed. This interval also corresponds to low solar activity, which leads to lower levels of GMA measured by Ap value.

As calculated, the correlation between the yearly profiles of the DHAI and the DHAG values is high $(r=0.7, P<$ $0.000001)$. There are significant seasonal differences in both the DHAI and DHAG levels too, as they are significantly higher during winter and spring in comparison with summer and autumn $(P<0.0009$ and $P<0.05$ for DHAI and DHAG, resp.). Meanwhile, the DHAI and DHAG values for summer and autumn are comparable (the same is true for winter and spring).

The yearly dynamics of DHAI and DHAG revealed the pronounced correlation with $\mathrm{WV}(P<0.00001)$, which was higher for the DHAI $(r=0.72)$ than for the DHAG $(r=0.49)$. This is due to more pronounced causal relationships between WV and APF in the I-range of their periods than in the G-range. However, the DHA versus Ap relationship is rather poor $(r=-0.16, P=0.003$ and $r=$ $-0.14, P=0.008$ for DHAI and DHAG, resp.).

3.2. Relation between EEI and Natural Physical Variables. The polynomial plot (Figure 1(a)) shows a nonlinear increase in the EEI number along all days of the interval studied if they are sorted by DHAI values in the ascending order (see also the plot of DHAI values, shown in a similar way in (Figure 1(b)). The EEI number proved to be significantly greater in the fourth DHAI quartile (with the highest DHAI values) in comparison with the other three quartiles ( $P$ is $0.0002,0.008$, and 0.02 for the first, second, and third quartile, resp.). The EEI numbers for these three quartiles themselves are comparable $(P>0.13)$. The threshold effect of DHAI was assumed to be realistic since the significant difference was found only for the boundary between the two groups of quartiles: the fourth one and the other three. Therefore, all days were divided into two categories, which were determined as low DHAI and high DHAI (Table 2).

After stepwise adjustments for the four potential confounding variables (ND, temperature, relative humidity, and atmospheric pressure), the EEI number remained significantly greater on days in the high DHAI-category when compared to days in the low DHAI-category (Table 2), indicating the independent contribution of high DHAI to the increased EEI number. However, the effect size (determined as the percentage of the relative EEI increase when comparing these two categories) of high DHAI decreased more than two times due to concomitant effects of these four variables. In fact, the effect size is $29.6 \%$ (95\% CI: 25.6 to 32.8 ) and $12.7 \%$ (8.3 to 16.4 ) for the raw and adjusted data, respectively.

The DHAG revealed no effects on the relation between EEI and DHAI. Actually, the high DHAI effect size after additional adjustment for the DHAG remained more or less the same with $11.7 \%$ (7.8 to 15.5 ).

When applying the same analysis to the DHAG variable, no significant relation between the EEI and DHAG was found for the raw data and the data adjusted for the four variables. However, the relation is apparent after the adjustment for either DHAI or for the four variables and DHAI. The corresponding plots for the EEI number adjusted for the DHAI or for the four variables and DHAI (as polynomial function) and the DHAG values obtained in a similar way (as in case for DHAI, Figures 1(a) and 1(b)) are presented in Figures 2(a) and 2(b), respectively. The EEI number is significantly less on days with high DHAG than on days with low DHAG (Table 2). The percentage decrease in the EEI number after adjustment for the four variables and DHAI is $-9.3 \%$ ( -7.2 to -11.7$)$.

Possible distorting influence of high DHAI on the EEI versus DHAG relation was proved by the repeated calculations after removing all days with high DHAI from the data. The significant decrease in the EEI number $(P<0.034)$ on days with high DHAG (CI: 2.94-3.85 Pa, $n 2=45$ ) versus days with low DHAG (CI: $1.73-1.84 \mathrm{~Pa}, n 1=209$ ) was validated even before adjusting for the DHAI. The effect size of high 
TABle 2: 95\% Confidence intervals (CI) for two categories of DHAI (low and high) values and corresponding EEI number for raw data (EEI/0), data adjusted for the four potential confounding variables (EEI/4), and then additionally adjusted for the DHAG (EEI/4G) (a) along with data on two categories of DHAG (low and high) values with corresponding EEI number for data adjusted for DHAI (EEI/I) and the five potential confounding variables including DHAI (EEI/4I) (b).

\begin{tabular}{|c|c|c|c|}
\hline \multicolumn{4}{|c|}{ (a) } \\
\hline Parameter & Low DHAI CI $(n 1=254)$ & High DHAI CI $(n 2=91)$ & $P$ value \\
\hline DHAI (Pa) & $1.81-2.00$ & $5.15-5.85$ & \\
\hline $\mathrm{EEI} / 0$ & $3.83-4.48$ & $4.81-5.95$ & 0.0002 \\
\hline $\mathrm{EEI} / 4$ & $4.08-4.57$ & $4.42-5.32$ & 0.028 \\
\hline $\mathrm{EEI} / 4 \mathrm{G}$ & $4.10-4.57$ & $4.42-5.28$ & 0.032 \\
\hline \multicolumn{4}{|c|}{ (b) } \\
\hline Parameter & Low DHAG CI $(n 1=214)$ & High DHAG CI $(n 2=131)$ & $P$ value \\
\hline DHAG $(\mathrm{Pa})$ & $1.73-1.82$ & $3.18-3.58$ & \\
\hline $\mathrm{EEI} / \mathrm{I}$ & $4.36-5.01$ & $3.70-4.53$ & 0.039 \\
\hline $\mathrm{EEI} / 4 \mathrm{I}$ & $4.37-4.89$ & $3.86-4.54$ & 0.032 \\
\hline
\end{tabular}

$P$ - significance of the difference in the EEI number between two categories (low and high) of DHAI and DHAG values.

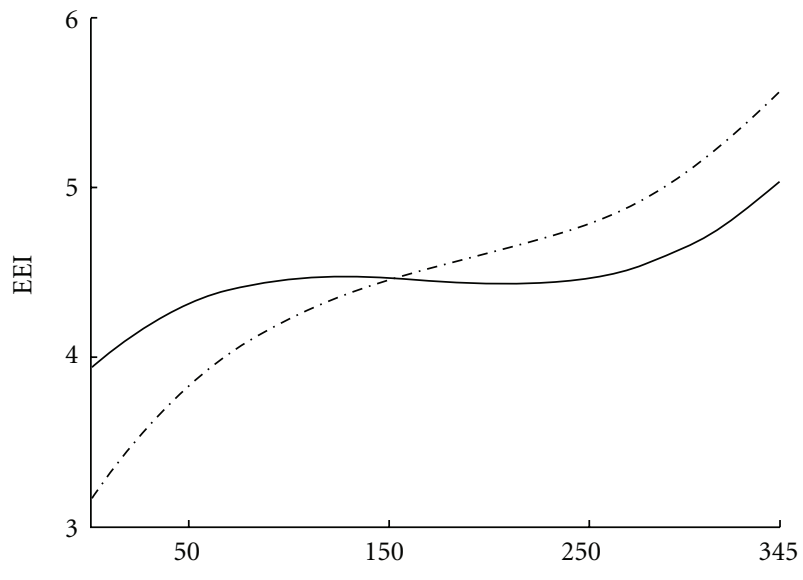

(a)

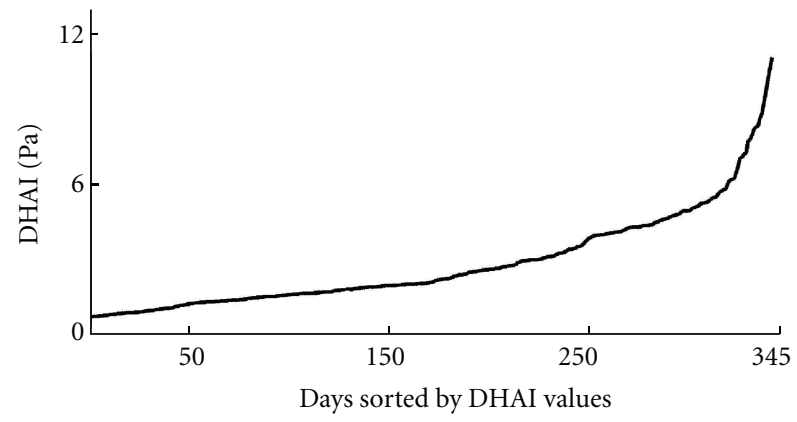

(b)

FIGURE 1: The EEI number (polynomial models) for raw data (dash-dot line) and data adjusted (solid line) for the four potential confounding variables (a), and the DHAI value (b) plotted against the days sorted by DHAI values in ascending order.

DHAG after adjustment for the four variables and DHAI is $-15.7 \%(-9.7$ to -22.7$)$. It seems that the high DHAG is favourable for the decrease in EEI number on days with low DHAI (not on days when high DHAI negative effects are strong and dominate).

On days with the high DHAI, the WV values were also profoundly high (CI: $3.02-3.38 \mathrm{~m} / \mathrm{s}, n 2=91)$ when comparing with those (CI: $1.83-2.04 \mathrm{~m} / \mathrm{s}, n 1=254, P<0.000001$ ) on days with the low DHAI. Therefore, the increased EEI number can be related to the high WV as well as to the high DHAI. In fact, this increase proves to be insignificant $(P>$ 0.13 ) after adjustment for the $\mathrm{WV}$, indicating the association between the WV and the high DHAI effects. These findings are in line with the high correlation between these two atmospheric variables due to their causal relationships, mentioned above.

A significant, though less pronounced correlation between the DHAG and WV is also mentioned above. However, the effect of high DHAG (when adjusting for the high
DHAI) proves to be independent on the $\mathrm{WV}$, since it remains significant $(P<0.01)$ after adjustment for the WV.

The same analysis as for the DHA was applied to the Ap variable, and this revealed that the relation between the EEI and Ap is also non-linear implying the threshold effect of higher Ap values. A significant difference in the EEI number is revealed between two categories of Ap values. The first category (CI: $5.00-5.65, n 1=253$ ) corresponds to the two lowest degrees of GMA (quiet and unsettled), with all Ap values being lesser 12. Therefore, this category is defined as low Ap. Such values prevail covering about $3 / 4$ of the interval analysed. The Ap higher values in the second category (CI: $19.40-26.25, n 2=92$ ) correspond mainly to the third degree of GMA (active geomagnetic conditions), which do not exceed 30. Since this value is generally accepted as moderate activity [15], this category is in fact of moderate Ap. Nevertheless, some higher values, (mentioned above) including two greatest magnitudes (101 and 102) slightly exceeding the degree of major storm conditions, are in this 


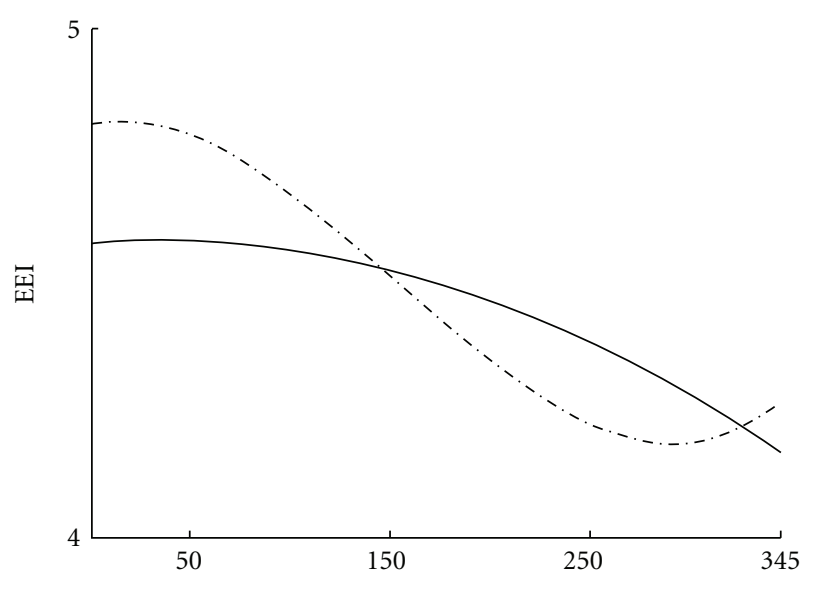

(a)

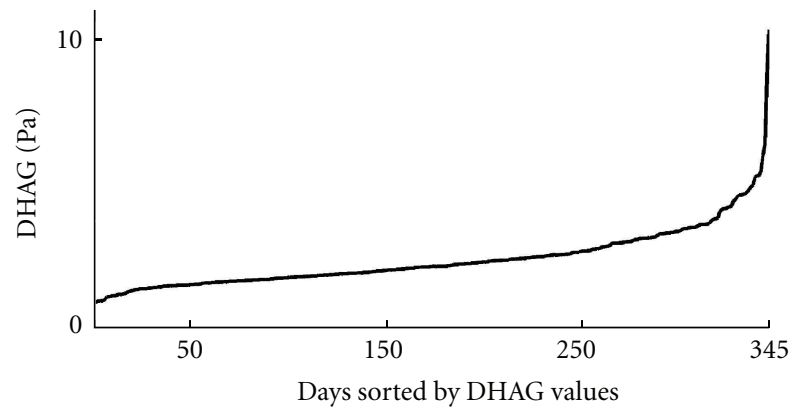

(b)

Figure 2: The EEI number (polynomial models) for data adjusted (dash-dot line) for the DHAI and data adjusted (solid line) for the four potentially confounding variables and DHAI (a), and the DHAG value (b) plotted against the days sorted by DHAG values in ascending order.

category. The effect of moderate Ap on the EEI number turns to be of opposite sense to the effect of high DHAI. On the other hand, it is similar to the effect of high DHAG. For the raw data, the EEI number significantly decreased (CI: 3.344.43, $P=0.013$ ) on days with moderate Ap in comparison with those (CI: 4.36-5.03) on days with low Ap, with the effect size of moderate Ap being $-17.4 \%$ ( -11.9 to -23.4). This decrease can be associated with the concomitant effects of the four potential confounding variables as it was more significantly pronounced for the raw data than after adjustment for these four variables $(P=0.037$, effect size: $-10.0 \%$ ( -6.4 to -13.9$))$.

As to the possible combined effects of DHA and Ap variables, the high DHAI $(n=91)$ and the high DHAG $(n=$ 131) levels were more frequently observed in combination with the low Ap values ( $n=76$ and $n=102$, resp.). Meanwhile, the moderate Ap values $(n=92)$ were mostly accompanied by the low DHAI and DHAG $(n=77$ and $n=63$, resp.). Weak inverse correlation between Ap and DHAG as well as between Ap and DHAI is mentioned above.

According to these combinations, the increase in EEI number revealed on days with high DHAI could be related to combined effects of high DHAI and low Ap. By the same token, combined effects of the moderate Ap and low DHAI could be responsible for the decrease in EEI number observed on days with moderate Ap. Actually, these combined effects are confirmed by the facts that the increase in the EEI number on days with high DHAI after additional adjustment for the Ap, as well as the EEI number decrease on days with moderate Ap after additional adjustment for the DHAI, becomes less significant $(P=0.047$ and $P=0.045$, resp.). The greater increase in the EEI number is shown on days with simultaneously high DHAI and low Ap, especially when comparing to the combination of moderate Ap and low DHAI $(n=78, P<0.005)$. On such days, the percentage increase is $20.2 \%$ (20.0 to 20.9) after adjustment for the four variables and the DHAG.
Meanwhile, high DHAG and moderate Ap, which both promoted the decrease in the EEI number, were registered mostly on separate days. Therefore, this decrease proves to be no less significant on days with moderate Ap after additional adjustment for the DHAG $(P<0.026)$, as well as on days with high DHAG after additional adjustment for the Ap (when controlling high DHAI effects, $P<0.012$ ) indicating independent on each other effects of these two variables.

\section{Discussion}

The results of this study provide new evidence on meterotropic effects of high APF in the far infrasound frequency range, namely, their relevance as to the increased risk of sport injuries. Moreover, new information on human sensitivity to APF with periods of internal gravity waves (G-range) is obtained using the database on sport injuries.

According to our analysis, it is reasonable to divide all days of the one-year interval studied into two categories of low and high DHAI, their subintervals of occurrence being about $3 / 4$ and $1 / 4$ of all days considered, respectively. The increased EEI number was documented for high DHAI. However, in spite of the quite wide range of low DHAI values, the EEI number persists to be low without significant changing, which indicates the adequate adaptation of people to such low APF levels in the I-range as to usual and common atmospheric noise. Meanwhile, the high APF in the Irange is a rather strong atmospheric factor resulting in the additional strain for the human adaptation mechanisms. As a consequence, a failure of the adequate behavioural reaction during sport activity is likely to take place, which leads, for example, to the increased EEI number. The non-linear relation between the EEI and DHAI manifested by the threshold effect for high DHAI is apparent. Similar relation was found between the DHAI and emergency transport events coded as circulatory system diseases in a previous study [3]. Hence, the high DHAI seems to be some kind of a predictor of 
the increase in the emergency events due to injury as well as circulatory system diseases.

The EEI versus DHAG relationship proved to be opposite to that of EEI versus DHAI. Such a peculiarity of effects for two frequency ranges is likely linked to the frequencydependent human sensitivity, resulting in the distinctive response. According to this, the APF with larger periods as in the G-range is rather a weaker physical factor than APF in the I-range with the same amplitude. It appears that higher DHAG have the activating and mobilizing effects leading to a more successful sport performance and as a consequence the decreased EEI number takes place. However, the high DHAG favourable effect fails to occur under high DHAI conditions when mechanisms of adaptation are already overstrained by this additional physical environmental factor. As revealed, the high DHAI effect prevails and even distorts the EEI versus DHAG relationship. That is the reason why the high DHAG effect is identified only for days with low DHAI or after adjustment for the DHAI variable.

Of interest are also the possible associations of the APF effects on the EEI number with WV, since the strong wind turbulence is the main source of APF. The mechanisms of the strong wind influence on human health and behaviour are not known. Some authors believe that adverse consequences of the strong wind on human health are due to negative effects of environmental concomitants such as ambient temperature, barometric pressure, positive ion concentrations, and so on [21]. Our analysis yield, the apparent relation of the high DHAI effects with the WV including the evident correspondence of the high DHAI with the high WV, which supports the suggestion that the high APFs are a general physical agent provoking biological response to these two atmospheric factors.

As to the human response, other natural physical environmental variables (e.g., variations of magnetic, electric and gravitational fields) can contribute to combined effects. Namely, the GMA variable related to human health parameters and behaviour is studied recently $[17,19,22]$. According to their inferences, the adaptation of healthy people to normal moderate GMA conditions is obvious, although extremely high or extremely low levels of GMA cause adverse effects on human beings. However, the combined effects of GMA and APF, which constitute changeable environmental background outdoors as well as indoors, have not been considered previously.

The interval studied corresponds to low solar activity, and, as can be expected, GMA is not pronounced. In fact, mainly days corresponding to the two lowest gradations of Ap values prevail. Meanwhile, the category of moderate Ap values corresponds mostly to the GMA third gradation identified as active geomagnetic condition $(16<\mathrm{Ap}<30)$. Such levels of GMA can be considered to be the favourable environmental factor from the viewpoint of an adequate adaptation of people (the decreased risk of sport injuries is indicated by the low EEI number). On the other hand, the very low GMA levels appear to be the rather unfavourable factor for human beings. Our analysis shows that the low GMA rather aggravates adverse effects of high APF. It seems that days with simultaneously high APF and low Ap are the most unfavourable, since the relative increase in the EEI number on these days was the highest.

According to a number of studies only extremely high GMA, such as severe magnetic storm conditions are the actual risk for adverse psychophysiological and autonomic reactions of healthy people. However, there was the lack of data on strong GMA levels in this study. Keeping in mind inhibiting effects of severe magnetic storms [22], it can be only speculated that simultaneous influence of high APF and severe magnetic storms are likely to enforce adverse effects on human health and behaviour. Hence the further investigations are needed using data within intervals of high solar activity, as well as data collected in the high-latitude regions where GMA conditions are most disturbed.

It is believed that natural APF could affect the human body through the ear [1]. Some authors suggested that the special area (viz., pars flaccida) in the tympanic membrane containing elastin fibers is actually a sensor mechanism for pressure fluctuations in the middle ear $[23,24]$. In favor of this view, the mechanical reactions of pars flaccida in response to very little changes in the middle ear pressure, as well as to slight pressure oscillations in far infrasound range, were demonstrated by the experimental studies on animals $[25,26]$.

We suggest that APF may influence the inner ear, too. The pressure transmission from the external ear canal to the inner ear is well studied in the otolaryngology. Particularly, it is established that a linear relationship exists between the amplitudes of pressure periodic changes in the inner ear and external ear canal of at least within the $+/-200 \mathrm{mmH}_{2} \mathrm{O}$ $(\approx 2000 \mathrm{~Pa})$ ranges [27]. Some authors believe that there is a pathway, through which changes in the pressure are transmitted from the middle ear to the inner ear and influence the activity of the otolithic receptors. This activity consequently affects the firing rates of the vestibular afferent fibers and of the neurons in the vestibular nucleus [28].

It is also shown that vestibular activity is dependent on the rates of ambient pressure changes in the middle ear. It is larger under higher rates of pressure changes [29]. May be different effects of high APF in two frequency ranges revealed in the present study are to some extent connected with this peculiarity of vestibular response.

Vestibular reactions to APF were suggested in previous experimental studies applying replicated barometric pressure oscillations in the infrasound frequency range. Kompanets [30] documented vestibulo-autonomic disorders and statokinetic instability in the population of aviators after onehour exposure to pressure oscillations in the barometric chamber. In line with this, a rise in heart rate in a group of healthy volunteers after 15-30 min exposure to simulated quasichaotic atmospheric pressure oscillations was reported in the following study [2]. It can be also suggested that the deterioration in attention parameters under exposition of pressure oscillations, revealed in both these studies, is linked with the influence of upward vestibular fibres on brainstem reticular activating systems and cortex.

The high APFs during adverse weather usually persist for many hours and even for some days. Such long-term influence of high APF on vestibular system can lead to autonomic 
and motor disorders and promote unsuccessful sports performance resulting in injury occurrences.

To conclude, the present study provides evidence that APF in the both frequency ranges is a poorly known contributor to human response to weather conditions. It appears that high DHAI is a strong meteorotropic factor associated with the increased EEI number, whereas the high DHAG levels are adequate for human adaptation. We propose that the opposite sense of the DHAI and DHAG relations with the EEI number revealed in the study is, at least partly, a result of dependency of human threshold sensitivity and adaptation ability on the APF frequency ranges.

The results obtained emphasize the importance of a more accurate and detailed analysis of the simultaneous and complex influence of meteorological and geophysical variables such as APF in both ranges of periods and GMA. Particularly, the combined effects of DHAI and DHAG modify the resulting output. Actually, the high DHAI unfavourable effects exert the distorting influence on the relation between the EEI and DHAG. Meanwhile, the low GMA aggravates the adverse effects of high DHAI promoting greater increase in the EEI number. According to our analysis the significant relationship exists between the strong wind and high DHAI effects, which is not the case for the high DHAG effects.

This is only exploratory study, limited by one-year period with low levels of GMA and by the geographical area (Kiev region) with moderate atmospheric conditions, where still or slightly windy weather prevails. The next step is to extend the analysis over a longer period including the time intervals with severe geomagnetic storm conditions and stronger atmospheric perturbations, as well as other geographical areas to receive data of high APF and stormy GMA levels.

\section{Acknowledgments}

This study was supported by the NATO Program Security Through Science, Collaborative Linkage Grant number 98376, funds from the University of Antwerp (BOF-NOI), and Ukrainian-Slovak Joint Research Project, number 70810. The authors thank A. V. Vershygora and V. T. Erygina (Kyiv Station of emergency services and medicine of catastrophes) for providing emergency transport events data.

\section{References}

[1] J. E. Green and F. Dunn, "Correlation of naturally occurring infrasonics and selected human behavior," Journal of the Acoustical Society of America, vol. 44, no. 5, pp. 1456-1457, 1968.

[2] A. Delyukov and L. Didyk, "The effects of extra-low-frequency atmospheric pressure oscillations on human mental activity," International Journal of Biometeorology, vol. 43, no. 1, pp. 3137, 1999.

[3] L. A. Didyk, Y. P. Gorgo, J. J. J. Dirckx et al., "Atmospheric pressure fluctuations in the far infrasound range and emergency transport events coded as circulatory system diseases," International Journal of Biometeorology, vol. 52, no. 7, pp. 553$561,2008$.

[4] E. E. Gossard and W. H. Hooke, Waves in the Atmosphere: Atmospheric Infrasound and Gravity Waves-Their Generation and Propagation, Elsevier, New York, NY, USA, 1975.
[5] J. I. Lumley and H. A. Panofsky, The Structure of Atmospheric Turbulence, Interscience Publishers, A division of John Wiley and Sons, London, UK, 1964.

[6] B. M. Vladymirskiy, "The atmospheric infrasound as a possible physical agent transferring solar activity influence to the biosphere," Problemy Kosmicheskoŭ Biologii, vol. 43, pp. 174179, 1982 (Russian).

[7] H. Richner and W. Graber, "The ability of non-classical meteorological parameters to penetrate into buildings," International Journal of Biometeorology, vol. 22, no. 4, pp. 242-248, 1978.

[8] P. G. Mezernitsky, Medical Meteorology. Brief Handbook, GIMK, Yalta, Crimea, 1934, (Russian).

[9] A. J. Bedard and T. M. Georges, "Atmospheric infrasound," Physics Today, vol. 53, no. 3, pp. 32-37, 2000.

[10] V. Čulić, N. Silić, and D. Mirić, "Triggering of ventricular ectopic beats by emotional, physical, and meteorologic stress: role of age, sex, medications, and chronic risk factors," Croatian Medical Journal, vol. 46, no. 6, pp. 894-906, 2005.

[11] F. G. Sulman, "Health, weather and climate," in Perspectives in Medicine, L. Reis van der, Ed., vol. 7, p. 166, Karger, Basel, Switzerland, 1976.

[12] P. L. von Deschwanden, "Influence of weather and climate on foehn and related diseases," in Progress in Human Biometeorology, Part II, S. W. Tromp, Ed., pp. 142-147, Swets and Zeitlinger Publishing Company, Lisse, The Netherlands, 1979.

[13] S. Rosen, Weathering. How the Atmosphere Conditions Your Body, Your Mind, Your Moods, and Your Health, M. Evans and Company, New York, NY, USA, 1979.

[14] A. J. Lee and W. M. Garraway, "The influence of environmental factors on rugby football injuries," Journal of Sports Sciences, vol. 18, no. 2, pp. 91-95, 2000.

[15] W. D. Parkinson, Introduction to Geomagnetism, Scottish Academic Press, London, UK, 1983.

[16] S. Dimitrova, "Different geomagnetic indices as an indicator for geo-effective solar storms and human physiological state," Journal of Atmospheric and Solar-Terrestrial Physics, vol. 70, no. 2-4, pp. 420-427, 2008.

[17] I. Stoilova and S. Dimitrova, "Geophysical variables and human health and behavior," Journal of Atmospheric and SolarTerrestrial Physics, vol. 70, no. 2-4, pp. 428-435, 2008.

[18] S. Oinuma, Y. Kubo, K. Otsuka et al., "Graded response of heart rate variability, associated with an alteration of geomagnetic activity in a subarctic area," Biomedicine and Pharmacotherapy, vol. 56, no. 2, supplement, pp. 284s-288s, 2002.

[19] E. Stoupel, "The effect of geomagnetic activity on cardiovascular parameters," Biomedicine and Pharmacotherapy, vol. 56, no. 2, supplement, pp. 247s-256s, 2002.

[20] R. McNamee, "Regression modelling and other methods to control confounding," Occupational and Environmental Medicine, vol. 62, no. 7, pp. 500-506, 2005.

[21] L. J. Cooke, M. S. Rose, and W. J. Becker, "Chinook winds and migraine headache," Neurology, vol. 54, no. 2, pp. 302-307, 2000.

[22] E. S. Babayev and A. A. Allahverdiyeva, "Effects of geomagnetic activity variations on the physiological and psychological state of functionally healthy humans: some results of Azerbaijani studies," Advances in Space Research, vol. 40, no. 12, pp. 1941-1951, 2007.

[23] L. E. Stenfors, B. Salen, and B. Winblad, "The role of the pars flaccida in the mechanics of the middle ear," Acta OtoLaryngologica, vol. 88, no. 5-6, pp. 395-400, 1979. 
[24] T. J. Rockley and W. M. Hawke, "The middle ear as a baroreceptor," Acta Oto-Laryngologica, vol. 112, no. 5, pp. 816-823, 1992.

[25] J. J. J. Dirckx, W. F. Decraemer, M. Von Unge, and C. Larsson, "Volume displacement of the gerbil eardrum pars flaccida as a function of middle ear pressure," Hearing Research, vol. 118, no. 1-2, pp. 35-46, 1998.

[26] L. A. Didyk, V. B. Bogdanov, V. A. Lysenko, N. P. Didyk, Y. P. Gorgo, and J. J. J. Dirckx, "The effects of slight pressure oscillations in the far infrasound frequency range on the pars flaccida in gerbil and rabbit ears," International Journal of Biometeorology, vol. 51, no. 3, pp. 221-231, 2007.

[27] K. Gyo, S. Nishihara, and N. Yanagihara, "Pressure transmission properties from the external ear canal to the inner ear. An experimental study using guinea pigs," Acta Oto-Laryngologica, vol. 106, no. 456, supplement, pp. 87-91, 1988.

[28] Y. Naito, J. Ito, J. Tsuji, and I. Honjo, "The influence of middle ear pressure on the otolith system in cats," Archives of OtoRhino-Laryngology, vol. 245, no. 5, pp. 321-324, 1988.

[29] M. Suzuki, H. Kitano, Y. Yazawa, and K. Kitajima, "The influence of rates of pressure change on pressure-induced vestibular response in guinea pigs," Journal of Otolaryngology of Japan, vol. 98, no. 5, pp. 820-824, 1995 (Japanese).

[30] V. S. Kompanets, "Effects of repeated opposite directional changes in the barometric pressure on man," Voenno-Medicinsky zhurnal, vol. 6, pp. 61-63, 1968 (Russian). 

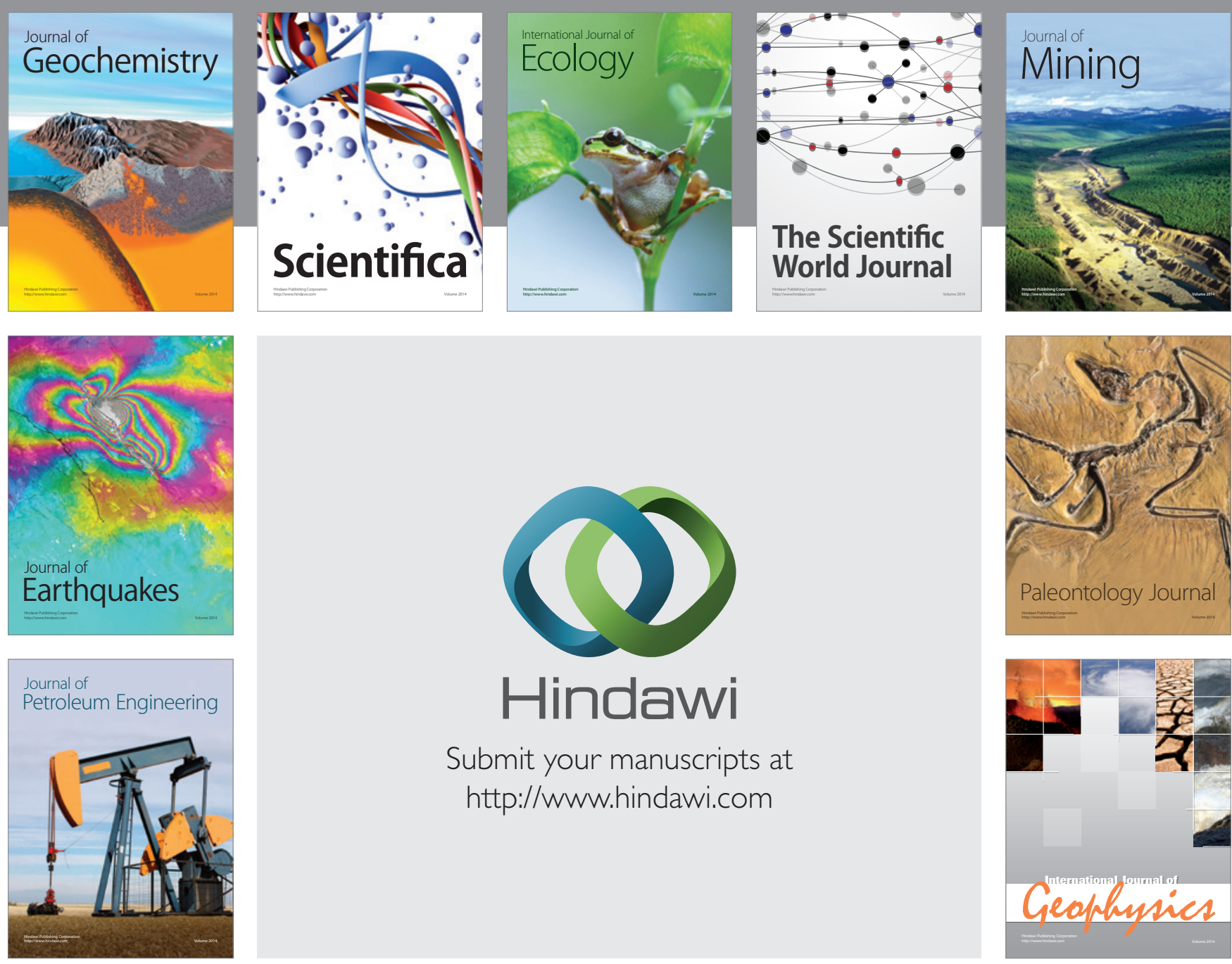

Submit your manuscripts at

http://www.hindawi.com
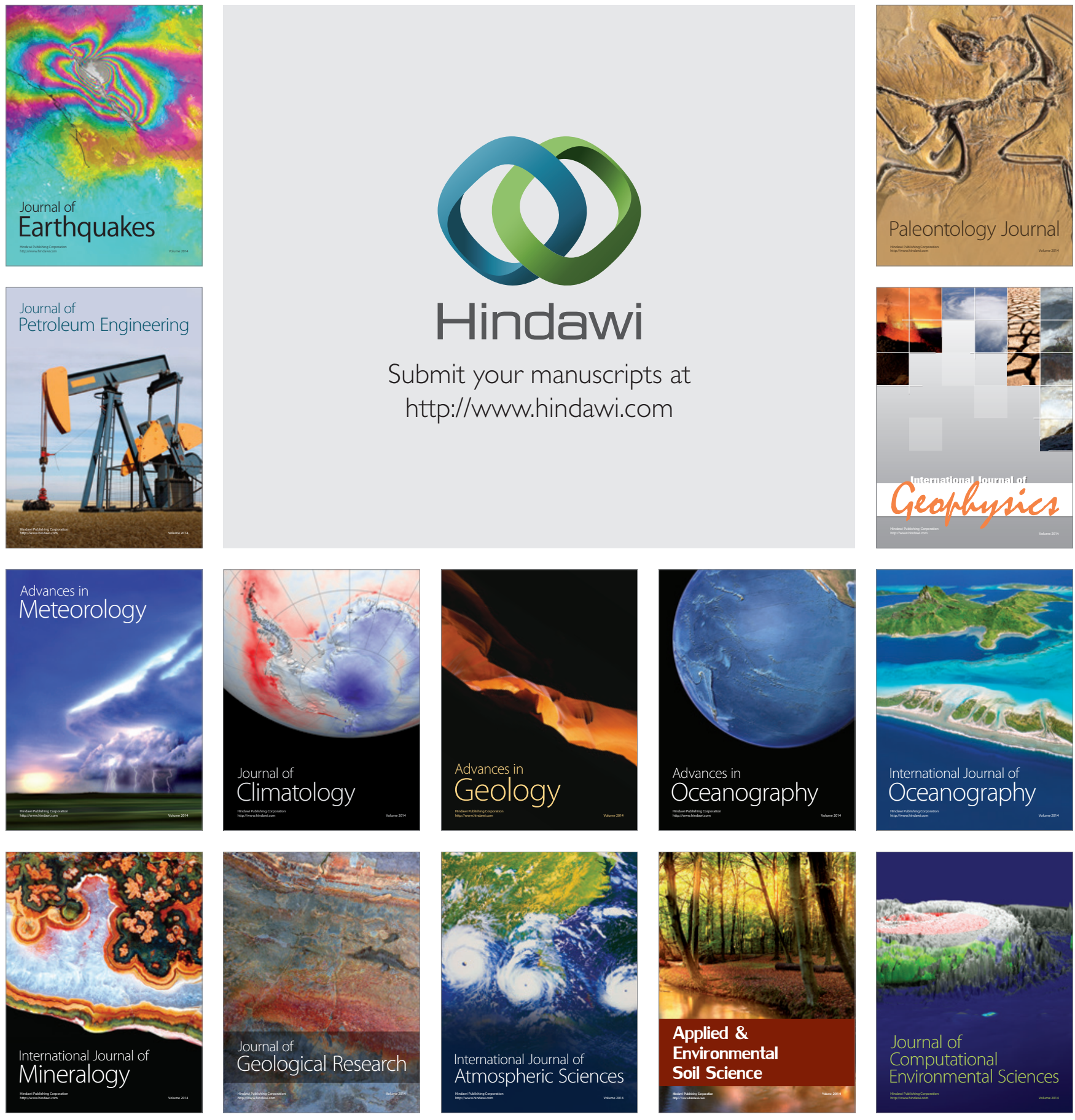